\title{
Intraoperative Spinal Sonography in the Evaluation of Intramedullary Tumors
}

\author{
Joel F. Platt, MD*, Jonathan M. Rubin, MD, PhD*, William F. Chandler, MD \\ Richard A. Bowerman, MD*, Michael A. DiPietro, MD*
}

Seventeen intraoperative spinal sonographic examinations were performed in 14 patients with intramedullary spinal cord neoplasms. Results of the ultrasound exams were correlated with preoperative imaging studies, surgical findings, and histopathologic analysis of the tumors.

Intraoperative spinal sonography accurately localized the intramedullary tumors, often revealing the need for extension of the initial laminectomy. The neoplasms appeared as expansile echogenic masses, with cystic com- ponents in over half. Ultrasound was as accurate as preoperative imaging in the evaluation of solid neoplasms, and was superior to computed tomography (CT) and magnetic resonance imaging (MRI) for delineating the cystic components of neoplasms. Intraoperative sonography should be used routinely in all surgical cases of intramedullary spinal cord neoplasms. KEY wORDS: neurosurgical ultrasound; intraoperative spinal sonography; spinal cord neoplasms. (J Ultrasound Med 7:317, 1988)
I ntraoperative spinal sonography (IOSS) is becoming widely accepted as a valuable aid in the intraoperative evaluation of a variety of intraspinal abnormalities. ${ }^{1-10}$ Published data on IOSS evaluation of intramedullary spinal cord neoplasms is somewhat limited when compared to reports of other types of spinal cord pathology. ${ }^{1-6}$ Most previous reports on the evaluation of spinal cord neoplasms with intraoperative sonography are limited to smaller patient populations. We report our experience with 17 intraoperative sonographic examinations in 14 patients with intramedullary spinal cord tumors. We attempt to better define the role and value of IOSS by describing the benefits and potential pitfalls that may be encountered in these examinations.

Received August 10, 1987, from the Departments of *Radiology and †Neurosurgery, University of Michigan Medical Center, Ann Arbor, Michigan. Revised manuscript accepted for publication November 24, 1987.

Address correspondence and reprint requests to Dr. Platt: Department of Radiology, Box 0030, University of Michigan Hospital, 1500 East Medical Center Drive, Ann Arbor, MI 48109-0030.

\section{MATERIALS AND METHODS}

Fourteen patients (ten women, four men; age range, 7 to 70 years) underwent 17 IOSS examinations. Examinations were performed with an ATL NeurosectOR portable real-time unit (Advanced Technology Laboratories, Bellevue, WA) with an in-line scan head capable of imaging at 3,5, or $7.5 \mathrm{MHz}$ transducer frequency. All scans in our series were performed at $7.5 \mathrm{MHz}$. Before sonography, the scan head was draped as previously described.?

The sonographic exams were performed via a posterior laminectomy with the patient lying prone. The laminectomy wound was filled with sterile saline to provide a fluid path for scanning. Initial sonographic evaluation was provided prior to opening the dura mater. Further use of sonography depended on the nature of the spinal surgery and on the neoplasm encountered. Scanning was performed in both longitudinal and transverse planes. Images were recorded on video tape and later photographed for the permanent patient record. Correlation of the IOSS exams with the operative

C 1988 by the American Institute of Ultrasound in Medicine $\bullet$ J Ultrasound Med 7:317-325, 1988 • 0278-4297/88/\$3.50 
findings, pathologic findings, and preoperative imaging was performed retrospectively.

\section{RESULTS}

The spinal cord neoplasms imaged in our series are summarized in Table 1 . The most common lesion encountered was an astrocytoma (five cases). Tumors occurred in all portions of the spinal canal (Table 2), but were most common in the thoracic or cervical thoracic region (nine cases).

Certain benefits of IOSS were evident in virtually all cases: localization, delineation of extent, and cystic vs. solid nature of the neoplasm.

Accurate localization of the intramedullary neoplasm before dural opening proved to be a useful feature of IOSS in all cases. In nine of our cases sonography demonstrated the need to extend the laminectomy.

Intraoperative spinal sonography delineated tumor extent by identifying zones of transition between normal and abnormal spinal cord based on definition of margins, and presence or absence of the central canal echo (Fig. 1). In 11 of our 14 patients good tumor border definition was present. In six of these 11 well-delineated neoplasms the surgeon could define margins as well, and a total resection was attempted. In the other five tumors with sonographically well-defined margins a total resection could not be performed, most commonly due to the long segment of tumor involvement.

In only three instances could IOSS not define tumor borders. One patient had a diffuse primitive neuroectodermal tumor involving the entire length of the spinal
Table 1: Types of Neoplasms Examined With Intraoperative Spinal Sonography

\begin{tabular}{lc}
\hline \multicolumn{1}{c}{ Spinal Cord Neoplasm } & No. of Patients \\
\hline Astrocytoma & 5 \\
Ependymoma & 3 \\
Ependymoblastoma & 1 \\
Dermoid & 1 \\
Teratoma & 1 \\
Low grade glioma & 1 \\
Hemangioblastoma & 1 \\
Primitive neuroectodermal tumor & 1 \\
\hline
\end{tabular}

Table 2: Location of Neoplasms Examined With Intraoperative Spinal Sonography

\begin{tabular}{lc}
\hline Location of Neoplasm & No. of Patients \\
\hline Cervical & 2 \\
Cervicothoracic & 3 \\
Thoracic & 6 \\
Conus & 2 \\
Entire cord & 1 \\
\hline
\end{tabular}

cord. The second patient had an infiltrative astrocytoma that at surgery was determined to be nonresectable. Intraoperative spinal sonography in this patient accurately reflected the infiltrating nature of this neoplasm by the lack of well-defined tumor margins and an absent central canal echo.

The third case demonstrated the one pitfall in evaluation of tumor extent. The patient was studied twice in a 10-day period. Preoperative magnetic resonance (MR)
Figure 1 Identification of tumor margin. Sagittal IOSS image (patient's head is toward viewer's (eff) shows transition from expanded spinal cord due to echogenic tumor mass (T), to normal appearing spinal cord with visible central canal (arrows).

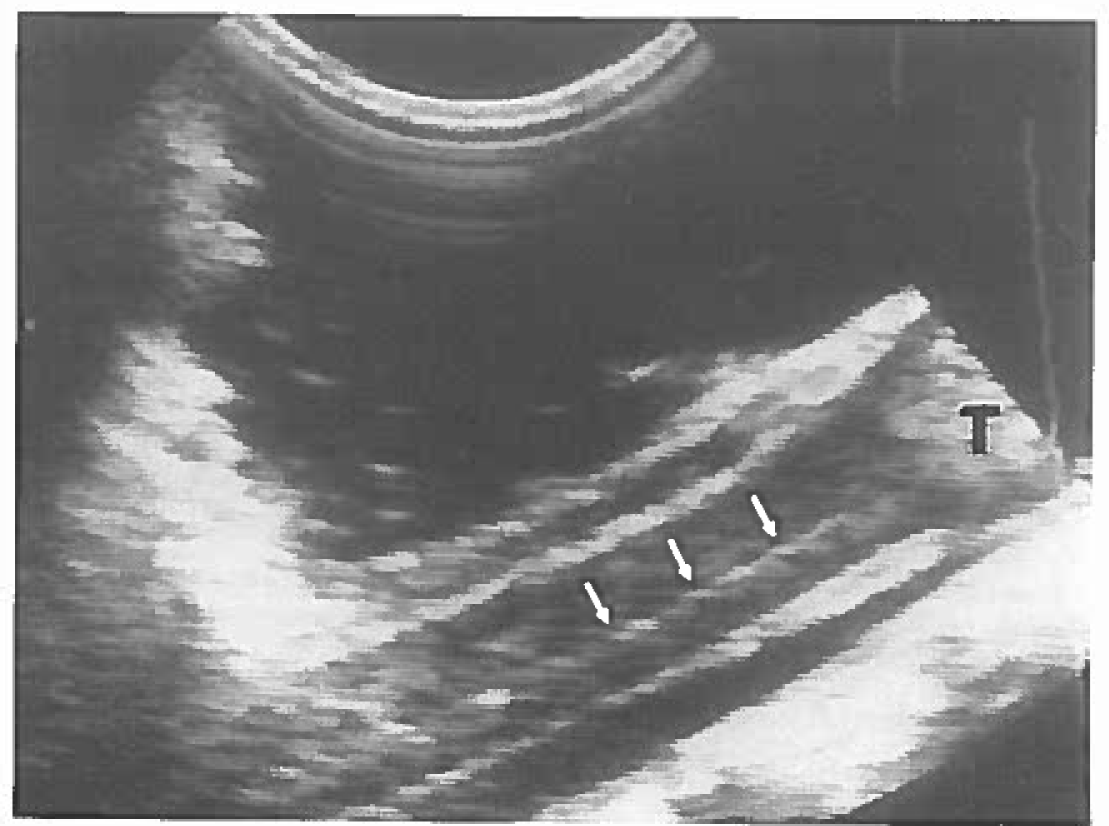



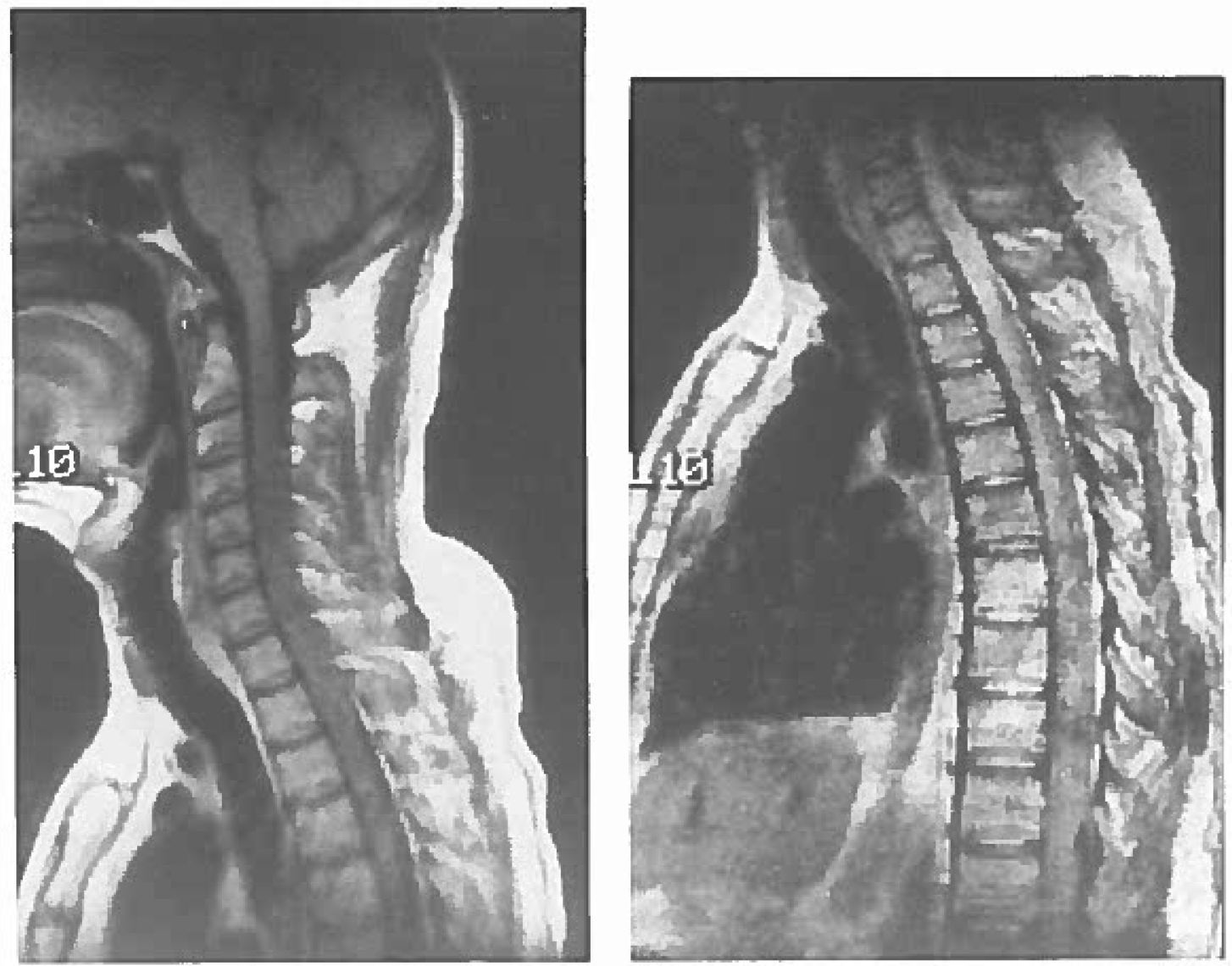

A

B

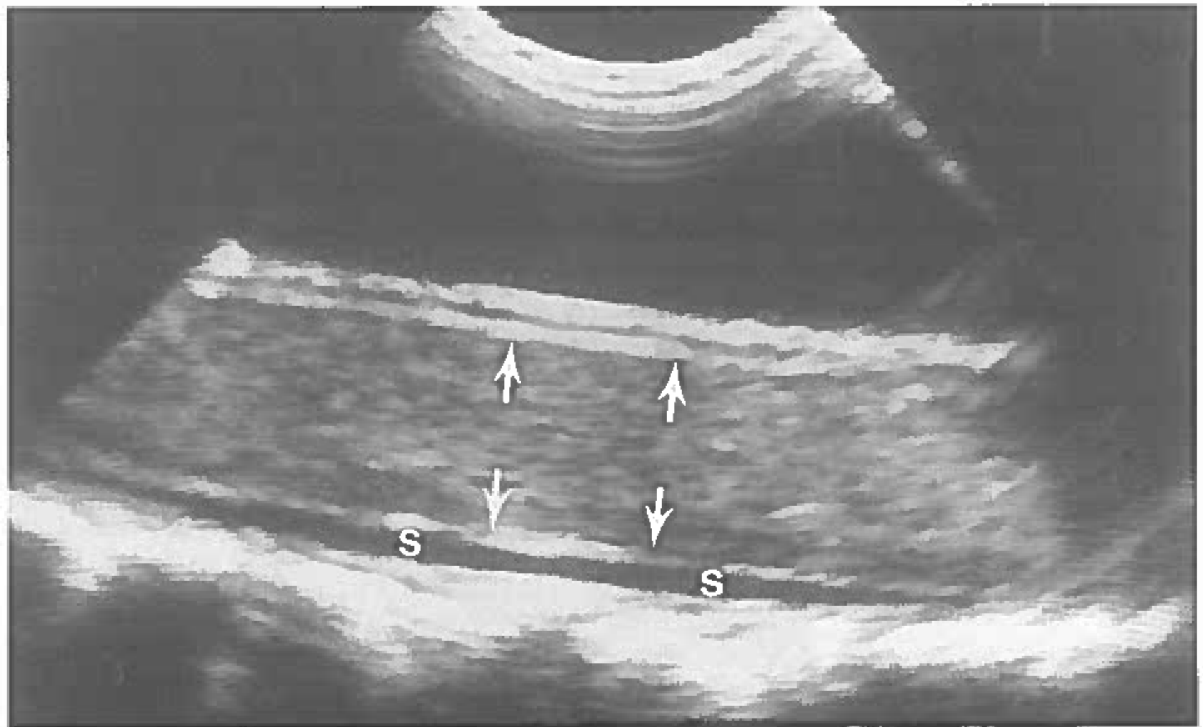

C

Figure 2 Ependymoma of spinal cord associated with cord edema, simulating a diffuse neoplasm. A, Sagittal T1-weighted image ( $T R=.30 \mathrm{TE} \equiv 30$ ) at $35 \mathrm{~T}$. Spinal cord is diffusely enlarged. B, Sagittal T2weighted image ( $\mathrm{TR}=1.50 \mathrm{TE}=40$ ). Diffuse intramedullary lesion of high signal intensity consistent with extensive neoplasm. C, Sagittal IOSS image (patient's head is toward viewer's left) from initial operation, shows diffuse widening of cervical spinal cord (arrows), absent central canal, and small amount of subarachnoid space anteriorly (S). 
Figure 2 (continued) D, Sagittal IOSS image (patient's head is toward viewer's left) from initial operation, demonstrates focal echogenic region (arrows) in

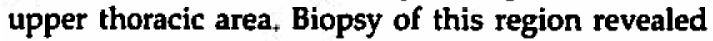
ependymoma. E, Sagittal IOSS image (patient's head is toward viewer's left) from reoperation 10 days later, shows local area of fluid within previous biopsy site (F), echogenic focal tumor (T) and normal appearing cervical spinal cord with central canal (arrows). F, Sagittal IOSS image (patient's head is toward viewer's $l e f t)$ from reoperation, demonstrates fluid within site of resected tumor (F), and normal spinal cord and central canal (arrows).

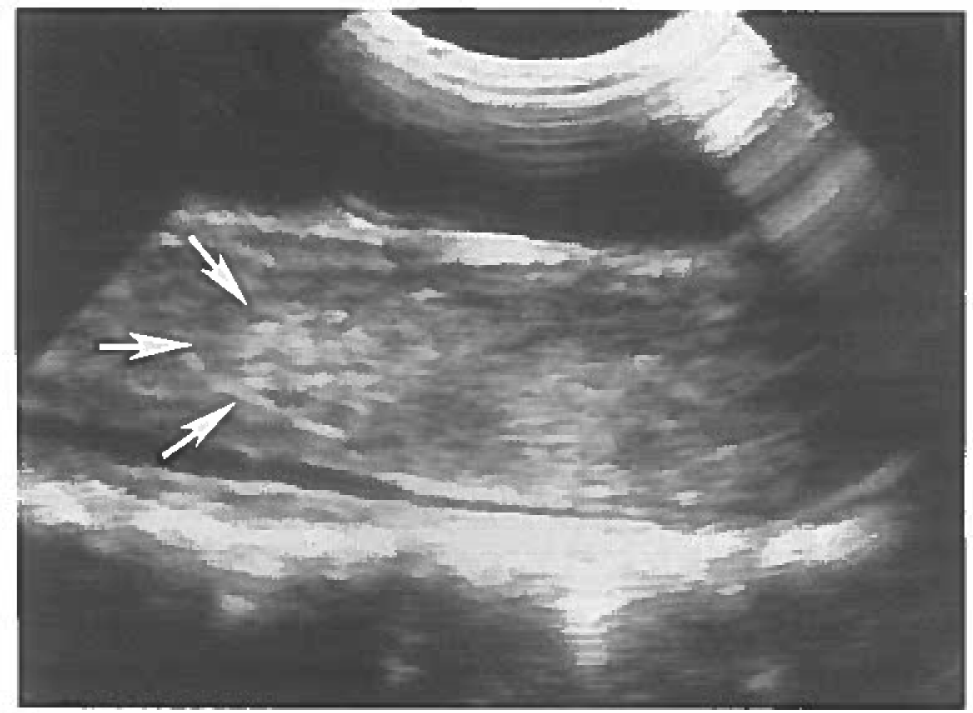

D

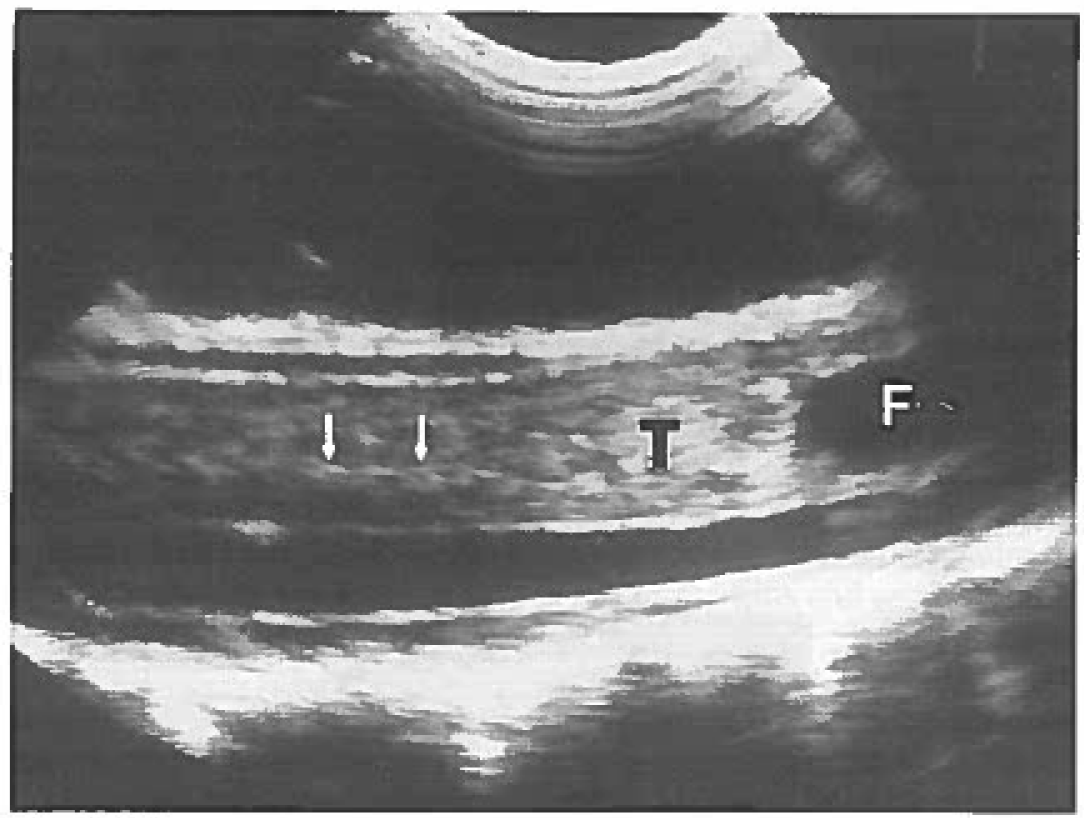

$\mathbf{E}$

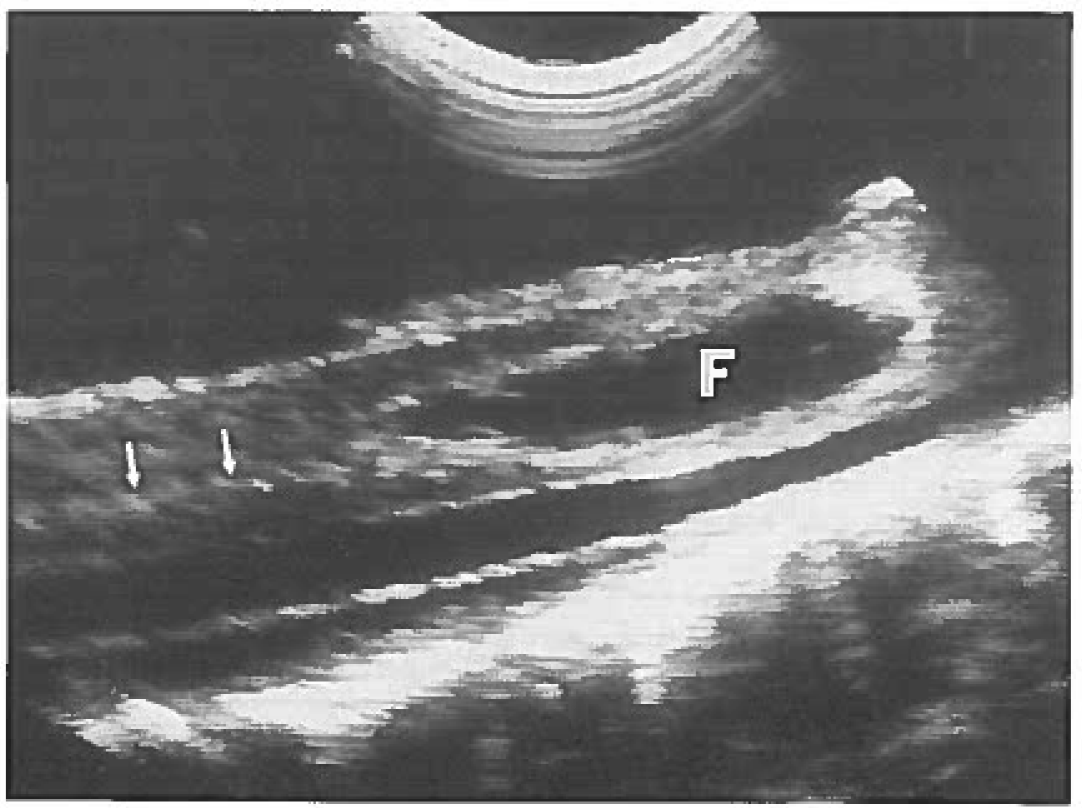




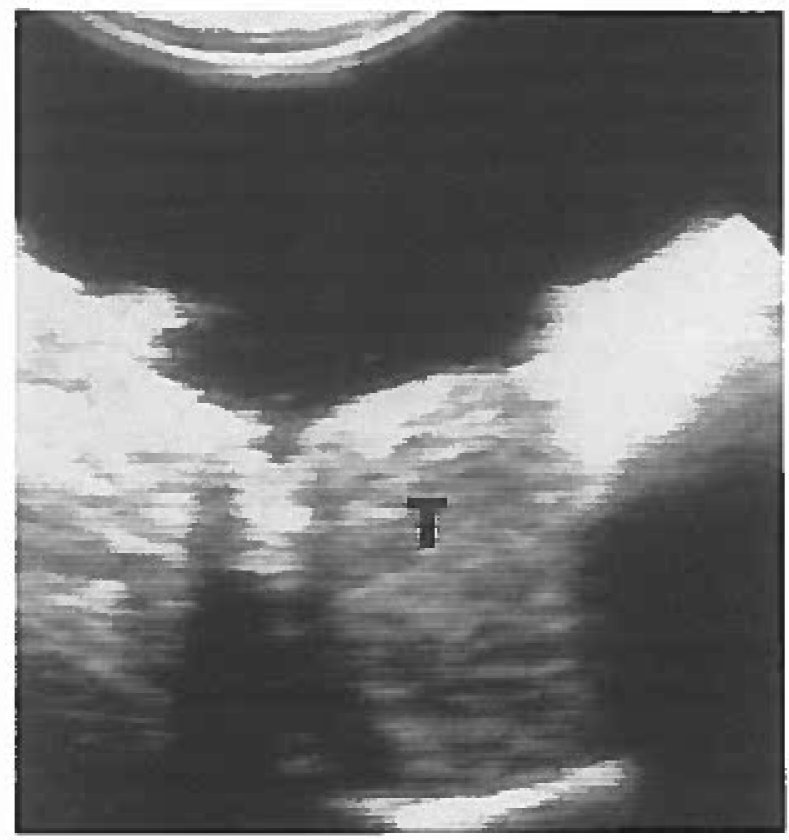

Figure 3 Cystic lesion appearing solid. Transverse IOSS image (patient's head is toward viewer's left) of cystic teratoma (T) which sonographically appeared solid.

exam revealed a diffuse abnormality of the cervicothoracic cord. Initial IOSS exam revealed a diffusely abnormal spinal cord with no recognizable tumor margins (Fig. 2). Biopsy of a more focally echogenic expanded area in the upper thoracic cord was consistent with an ependymoma, and a second operation was performed 10 days later for total tumor resection. At reoperation IOSS demonstrated focal echogenic residual neoplasm with well-defined tumor margins, a local area of fluid within the previous biopsy site, and normal appearing spinal cord adjacent to the neoplasm, with a clearly recognizable central canal echo (Fig. 2). Presumably the changes on the initial IOSS exam were secondary to cord edema, simulating the appearance of a diffuse extensive neoplasm.

Intraoperative spinal sonography was very accurate in characterizing the nature (solid/cystic) of intramedullary tumors. In only one case did ultrasound predict a solid lesion, when pathologic analysis revealed a cystic abnormality. This lesion, a teratoma, was composed of two cysts filled with thick creamy debris (Fig. 3). The lesion also had a solid appearance on enhanced computed tomography (CT) scanning.

Certain characteristics of neoplasms' solid components were invariably present. The neoplasms resulted in expansion of the cord, loss of the central canal echo, and increased echogenicity when compared to normal spinal cord tissue.

In general, it was impossible to specifically distinguish among different tumor cell types based on IOSS alone. In one case, a hemangioblastoma, distinctive features were noted that suggested the correct diagnosis. This partially cystic and solid tumor was associated with a large tortuous blood vessel with obvious flow during real-time examination (Fig. 4). These findings were not associated with any other neoplasm, and this appearance may be specific for hemangioblastoma. In this case, IOSS alerted the surgeon to the presence and location of the abnormal blood vessel, so that it could be avoided during biopsy and tumor resection.

Intraoperative spinal sonography was commonly used to guide the neurosurgeon in diagnostic tumor biopsy. This guidance was particularly useful in more difficult

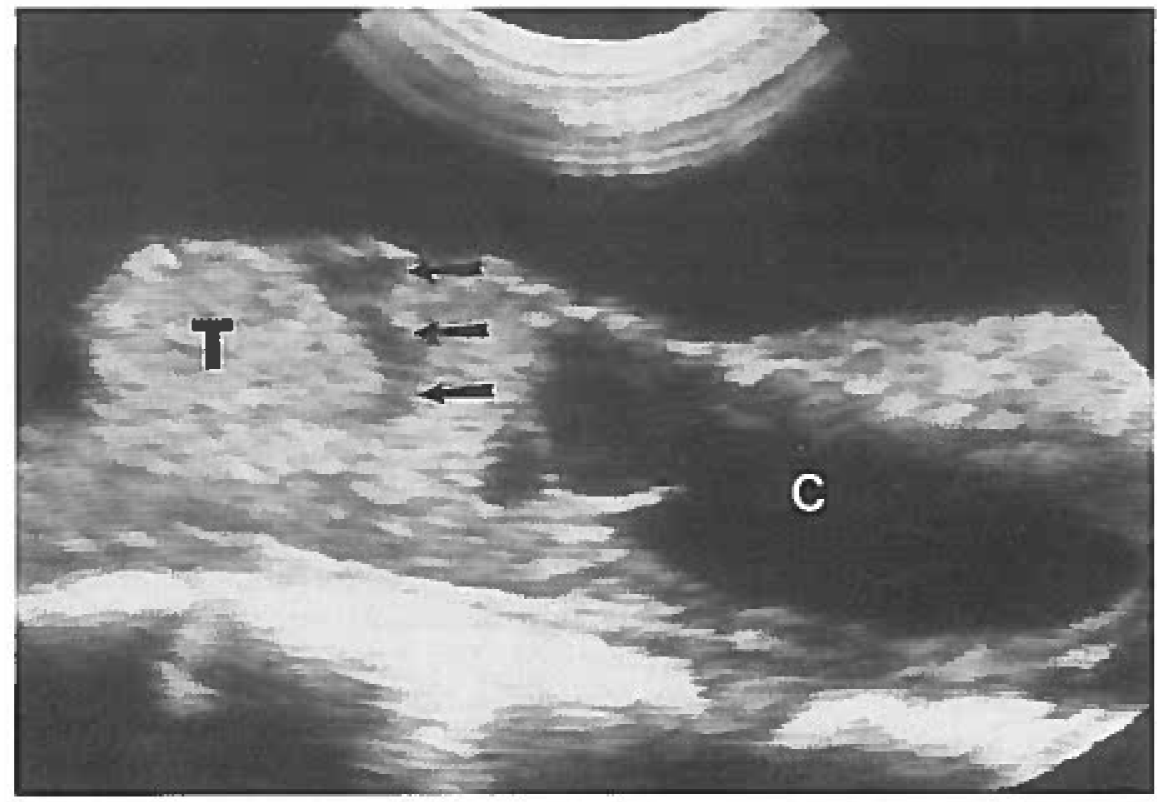

Figure 4 Hemangioblastoma of thoracic cord. Sagittal IOSS image (patient's head is toward viewer's left) of echogenic tumor ( $T$ ) and cyst $(C)$ associated with enlarged blood vessel (arrows) with obvious internal flow at real-time examination. 
Figure 5 Nontumoral cyst adjacent to cystic astrocy toma. A, Sagittal IOSS image (patient's head is toward viewer's left) of cystic intramedullary mass (M). B, Sagittal IOSS image just cephalad to cystic tumor reveals an extensive nontumoral cyst (C) which was subsequently opened and drained.

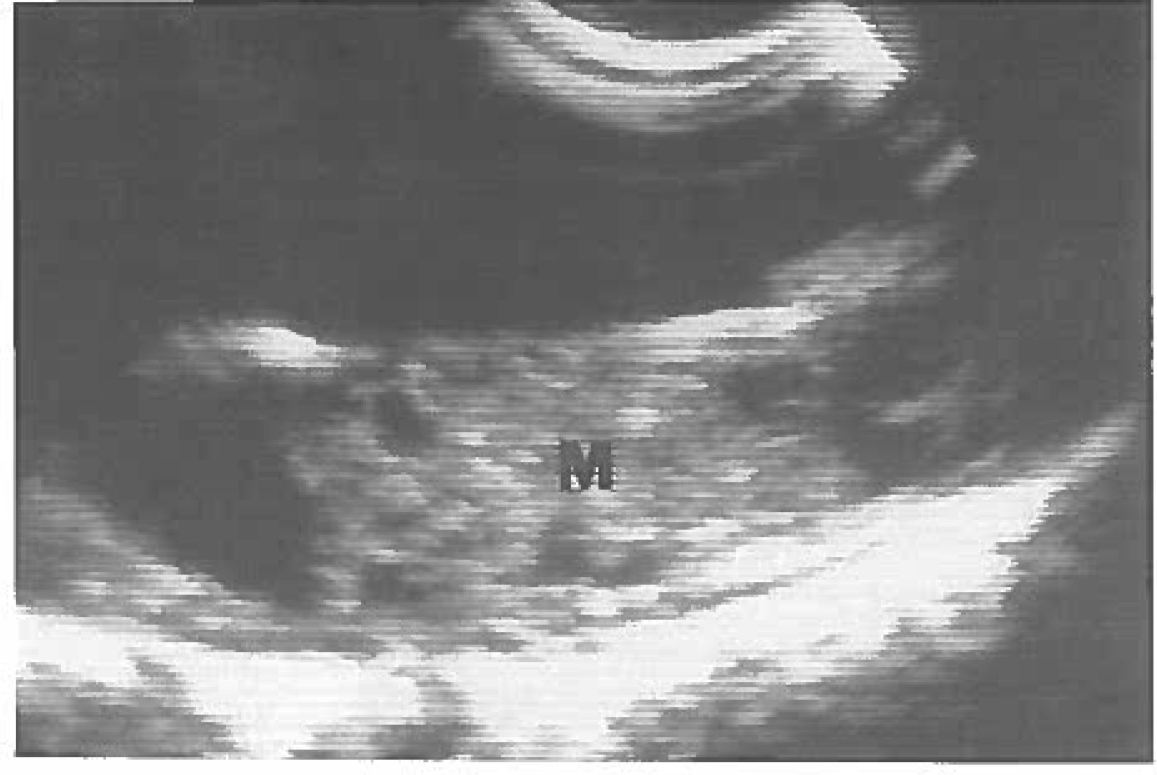

A

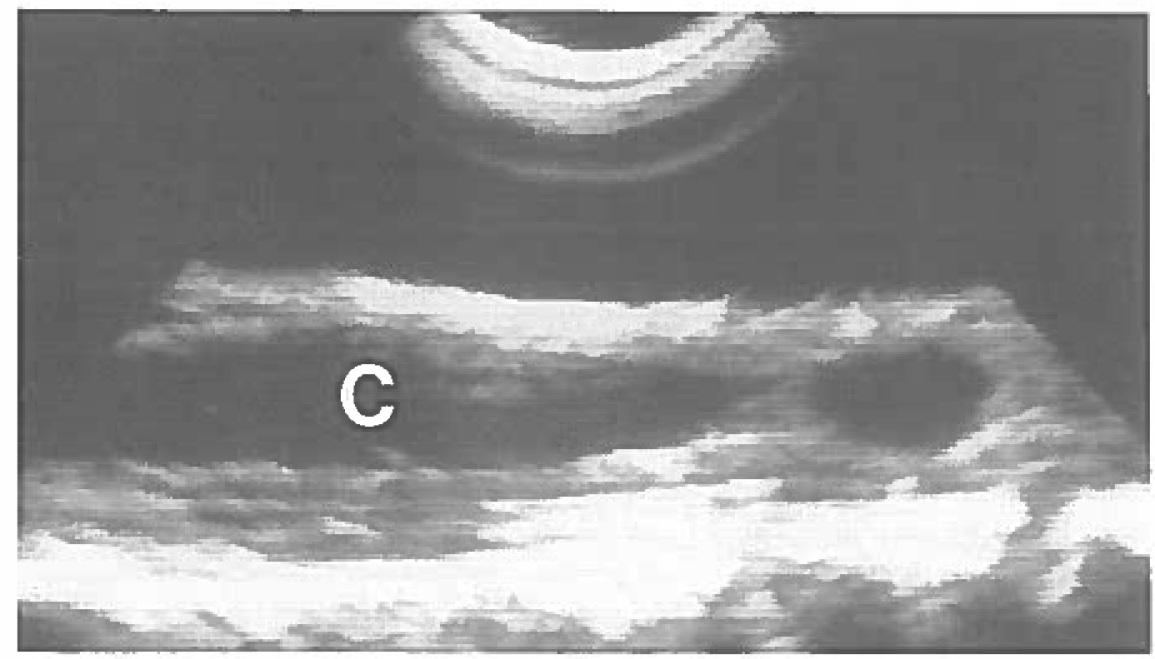

B

biopsies, such as in the case of the hemangioblastoma described above, or primarily cystic neoplasms. Intraoperative spinal sonography identified sites for obtaining adequate tissue, while at the same time minimizing possible spinal cord trauma.

Although no uniform type of treatment was used for the wide variety of neoplasms in our series, in at least four cases IOSS was used to monitor tumor debulking or to confirm gross tumor resection.

Nine of the 14 intramedullary neoplasms in our series were associated with intratumoral cystic components, ranging from a solitary large cyst to multiple tiny cystic regions, In five of these nine, IOSS provided additional information to the neurosurgeon not evident from preoperative studies. Intraoperative spinal sonography correctly delineated the cystic nature of three neoplasms thought to be primarily solid by preoperative imaging. In another case, IOSS revealed the absence of a large cystic component predicted to be present by preoperative magnetic resonance imaging (MRI). In the fifth case, sonography differentiated cystic neoplasm from an adjacent, nontumorous cystic abnormality (syrinx) (Fig. 5).

Five patients' IOSS examination revealed cystic areas not surrounded by solid neoplasm. These nontumorous cysts were opened and drained with ultrasound guidance. Intraoperative postintervention scanning was used to assess the adequacy of the drainage.

In one case, an extramedullary, septated, hypoechoic region was identified. The surgical dissection in this case proved difficult, and the unusual extramedullary mass, not depicted by MRI, likely represented arachnoiditis (Fig. 6). 


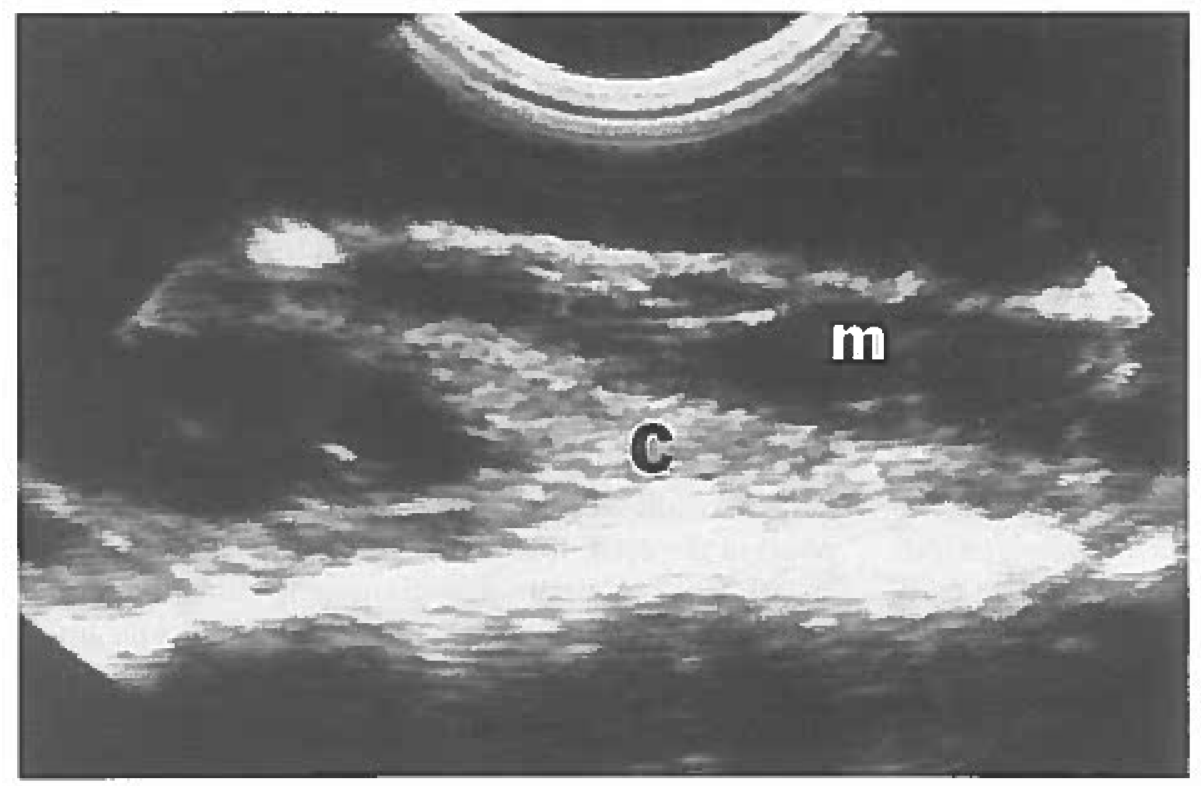

A

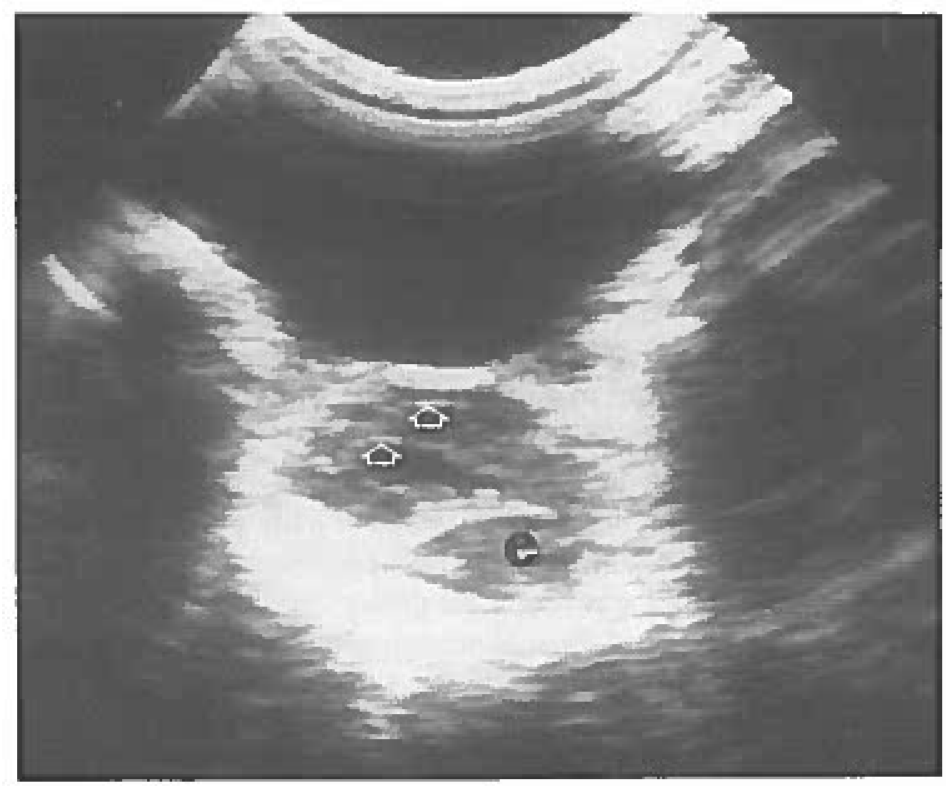

Figure 6 Arachonoiditis complicating surgery for intramedullary neoplasm. A, Sagittal IOSS image (patient's head is toward viewer's (eft) of hypoechoic septated mass-like area (M), with anterior displacement of spinal cord (C). B, Transverse IOSS image (patient's right is toward viewer's (eft) shows thickened septae (arrows) within the mass-like region. Displaced spinal cord $(\mathrm{C})$ is noted anteriorly to the left. This area was very difficult to dissect and was thought to be arachnoiditis at surgery.

B

\section{DISCUSSION}

Intraoperative spinal sonography is gaining greater acceptance as a valuable adjunct in surgery for a variety of abnormalities. ${ }^{1-10}$ Previous reports on the role of sonography in the evaluation of intramedullary tumors are limited by small patient groups. ${ }^{3,6}$ Our experience with a larger patient population with a wide variety of lesions reemphasizes many of the previously reported uses of IOSS, suggests additional benefits, and illustrates potential pitfalls.
Clearly IOSS is useful in the localization of intramedullary neoplasms before dural opening. In nine of our cases the initial sonographic examination revealed the need to extend the laminectomy either cranially or caudally. At the same time, it enabled the neurosurgeon to limit the laminectomy as much as possible, thereby decreasing the chance of progressive or long-term deformity of the spine.

A second definite benefit of JOSS was definition of tumor extent. This analysis was based on delineation of tumor margins and identification of the central canal 
echo. In 11 of our 14 patients good tumor border definition was present on the ultrasound exam. Subsequently, total tumor resection was attempted in six patients. However, sharp tumor margins do not necessarily indicate that a resection can be performed, because long segment tumors may be sharply delineated. Identification of tumor borders by lOSS, however, does indicate that complete resection can be considered unless contraindicated for other reasons. In two cases, no recognizable tumor border was evident by IOSS, which correlated well with the surgical-pathologic findings of extensive infiltrating neoplasm not amenable to surgical resection. We encountered only one case in which IOSS incorrectly depicted tumor extent. A focal ependymoma appeared as an extensive spinal cord tumor due to cord edema, which is echogenic similar to its appearance in the brain. "It is interesting to note that in a recent report edema was described as being hypoechoic. ${ }^{12}$ This appearance is atypical in the neural axis and is different from our experience. In that report, the authors claimed that ultrasound could distinguish between edema and tumor, where MR could not. ${ }^{12}$ Clearly, this distinction was not possible in our case.

A third clear benefit of IOSS is to identify and localize cystic and solid components of neoplasms. Poser, in an autopsy series, reported $31 \%$ of spinal intramedullary neoplasms were associated with cord cavitation (cysts). ${ }^{13}$ Sixty-four percent ( 9 of 14) of spinal cord neoplasms in our series were associated with cysts, similar to a recent report that found $53 \%$ of intramedullary tumors had cystic regions. ${ }^{6}$ Five of our neoplasms were associated with cord cavitation not completely surrounded by tumor, apparently representing nontumorous cysts. It is interesting to note that all five patients with nontumorous cysts had, in addition, a cystic component to their intramedullary neoplasms. No totally solid tumor was associated with a nontumorous type cyst. This observation suggests that a more thorough search for cystic regions, cephalad and caudal to the primary neoplasm, should be performed in patients with cystic neoplasms. A wide variety of cystic abnormalities, ranging from a solitary large cyst to innumerable tiny cystic regions, were associated with the tumors in our series. Fifty-six percent (5 of 9) of the cystic neoplasms in our series, were more accurately characterized by IOSS than preoperative imaging (CT or MRI). The most common benefit was delineation of the primarily cystic nature of a tumor thought to be solid preoperatively. In no case was MRI or CT better than IOSS at demonstrating the cystic components of neoplasms. The one primarily cystic tumor incorrectly characterized by IOSS was a teratoma. The echogenic appearing cysts were filled with thick, creamy fluid and desquamated tissue. This case points out a potential pitfall in sonographic identification of cystic structures. The cyst may be falsely deemed solid, if filled with material producing multiple reflective interfaces. This neoplasm was also incorrectly deemed solid by preoperative imaging studies.

A fourth benefit of lOSS is in the guidance and monitoring of interventional procedures performed during surgery. Intraoperative spinal sonography frequently played a prominent role in guiding diagnostic tumor biopsies. Ultrasound guidance was invaluable in tumors where a specific site was required for biopsy, such as a tumor nodule or a cyst wall. Accurate guidance reduced the number of biopsies required, thereby minimizing possible complications. Intraoperative spinal sonography also allowed the neurosurgeon to avoid specific areas of the cord, such as the enlarged blood vessel seen in the case of a spinal hemangioblastoma. The other major procedure in which lOSS proved beneficial was aspiration or drainage of cystic structures. Three intratumoral cysts were successfully aspirated under ultrasound guidance, and aspiration of one cyst relieved the degree of cord swelling. All five patients with nontumoral cysts underwent sonographically guided opening and drainage of these cysts. Intraoperative spinal sonography guided cyst puncture confirmed proper needle position and monitored adequacy of drainage.

Previously published data indicate that intramedullary tumors exhibit only a slight difference in echogenicity compared to normal spinal cord tissue. ${ }^{3}$ The intramedullary tumors in our series, however, were invariably more echogenic than normal cord.

Prediction of exact tumor cell type based solely on IOSS could not in general be performed. One neoplasm did exhibit findings allowing for exact tumor diagnosis. This spinal hemangioblastoma was noted to contain an enlarged tortuous blood vessel, with obvious flow at real-time examination. The appearance of spinal hemangioblastoma has been previously described. ${ }^{10}$

Intraoperative spinal sonography may also reveal unexpected findings not evident in preoperative studies. This benefit was seen in one case in which the intramedullary neoplasm was associated with a septated extramedullary mass. The spinal cord was displaced anteriorly as demonstrated sonographically. Surgical dissection in this case was difficult, and the mass was thought to be secondary to arachnoiditis. It was not evident on preoperative MRI.

\section{REFERENCES}

1. Knake JE, Chandler WF, McGillicuddy JE, et al: Intraoperative sonography of intraspinal tumors: initial experience. AJNR 4:1199, 1983

2 Dohrmann GJ, Rubin JM: Intraoperative ultrasound imaging of the spinal cord syringomyelia, cysts, and tumors - a preliminary report. Surg Neurol 18:395, 1982 
3. Quencer RM, Montalvo BM, Green BA, et al: Intraoperative spinal sonography of soft-tissue masses of the spinal cord and spinal canal. AJR 143:1307, 1984

4. Hutchins WM, Vogelzang RL, Neiman HL, et al: Differentiation of tumor from syringohydromyelia: intraoperative neurosonography of the spinal cord. Radiology 151:171, 1984

5. Enzmann DR, Irwin KM, Silverberg GD, et al: Spinal cord tumor imaging with CT and sonography. AJNR 6:95, 1985

6. Goy AM, Pinto RS, Raghavendra BN, et al: Intramedullary spinal cord tumors: MR imaging, with emphasis on associated cysts. Radiology 161:381, 1986

7. Rubin JM, Dohrmann GJ: Work in progress. Intraoperative ultrasonography of the spine. Radiology 146:173, 1983

8. Quencer RM, Morse BMM, Green BA, et al: Intraoperative spinal sonography: adjunct to metrizamide CT in the as- sessment and surgical decompression of post-traumatic spinal cord cysts. AJNR 5:71, 1984; AJR 142:593, 1984

9. Knake JE, Gabrielsen TO, Chandler WF, et al: Real-time sonography during surgery. Radiology 151:461, 1984

10. Sanders WP, Ausman Jl, Dujouny $M$, et al: Ultrasonic features of two cases of spinal hemangioblastoma. Surg Neurol 26:453, 1986

11. Smith Sj, Vogelzang RL, Marzano MI, et alt Brain edema: ultrasound examination. Radiology 155:379, 1985

12. Post MJD, Quencer RM, Green BA, et al: Intramedullary spinal cord metastases, mainly of nonneurogenic origin. AJR 148:1015, 1987

13. Poser $\mathrm{CM}$ : The relationship between syringomyelia and neoplasm. In: American Lecture Series No. 262: American Lectures in Neurology. Springfield, IL, Thomas, 1956 